\title{
Free-Living Amoebae as Human Parasites and Hosts for Pathogenic Microorganisms ${ }^{\dagger}$
}

\author{
Patrick Scheid ${ }^{1,2}$ \\ 1 Department XXI Medical Microbiology, Laboratory of Medical Parasitology, Central Military Hospital \\ Koblenz, Andernacherstr. 100, 56070 Koblenz, Germany; patrickscheid@uni-koblenz.de \\ 2 Parasitology and Infection Biology Group, Department of Biology, Institute of Integrated Sciences, \\ University of Koblenz-Landau, Universitätsstr. 1, 56070 Koblenz, Germany \\ + Presented at the 3rd EWaS International Conference on "Insights on the Water-Energy-Food Nexus", \\ Lefkada Island, Greece, 27-30 June 2018.
}

Published: 31 July 2018

\begin{abstract}
Free-living amoebae (FLA) can be found both in natural aquatic environments and in artificial, man-made aquatic environments. For a long time, (naked) FLA were considered to be harmless protozoa of soil and water. However, research since the 1960s has demonstrated that FLA can be pathogenic to humans and animals with nearly $100 \%$ morbidity from some strains. As etiological agents of the so-called Acanthamoebiasis, the Acanthamoebae can trigger several specific diseases (or symptoms) in humans. The amoebiasis of the central nervous system is called granulomatous amebic encephalitis (GAE), when Acanthamoebae are the etiological agents, showing subacute to chronic progress. GAE differs clinically from the primary amoebic meningoencephalitis (PAM(E)), which is caused by Naegleria fowleri. The Acanthamoeba keratitis is not necessarily associated with an immune suppression, but rather with a trauma, exposure to contaminated water or, particularly, the improper handling of contact lenses, which promotes infection. The clinical picture of GAE by Balamuthia mandrillaris is characterized by headache and neck stiffness. The infection is chronic and the time between infection and appearance of neurological symptoms may range from one month to about two years. The prevalence of FLA in water networks is associated with biofilms, where the amoebae live within a biocoenosis sympatric with other microorganisms. These biofilms serve as feeding grounds for the FLA and provide protection to a certain degree while the FLA adhere to the surfaces. In such a biocoenosis there are multiple interactions between FLA and other microorganisms: In addition to their role as pathogens, FLA are known to serve as host of and vehicles for diverse intracellular organisms (bacteria, viruses, eucaryonts), some of them being natural human pathogens. They act as reservoir or vehicle for various microorganisms such as various Legionella sp., Listeria monocytogenes, Pseudomonas aeruginosa and some Mycobacterium sp. In the cyst-stage of the FLA these intracellular organisms (endocytobionts) are protected to a high degree against any adverse environment (FLA as "Trojan horse"). This host-endocytobiont relationship may further lead to health risks in terms of the development of pathogenicity/virulence and antibiotic resistance (FLA as "Trainings ground").
\end{abstract}

Keywords: Free-living amoebae; Acanthamoeba; Naegleria; vectors; reservoirs; endocytobionts; endosymbionts; GAE; PAM(E); Acanthamoeba keratitis

\section{Introduction}

Naked Free-living amoebae (FLA) are protozoa that are ubiquitously distributed in soil- and aquatic habitats and complete their entire life cycle without a host. They inhabit biocoenoses in natural and artificial humid environments. These artificial environments may include man-made (engineered) habitats including tap water systems (even in hospitals), cooling towers, water tanks, 
wastewater treatment plants, pharmaceutical factory drains, swimming pools, sewage, contact lens cases, air conditioning equipment, dialysis units, dental treatment units and others, are affected from FLA [1]. There they regularly live in biofilms sympatric with other microorganisms, resulting in interesting relationships. The biofilms occur in water supply lines/plumbings in industry, private houses, and even in healthcare areas [2]. Within these biofilms FLA and other microorganisms are protected to a certain degree against harsh conditions or countermeasures, e.g., disinfection procedures [3]. FLA are predatory heterotrophic microorganisms feeding as trophozoites on bacteria, cyanobacteria, fungi and algae while adhering to surfaces. While there are many studies and regulations involving several bacteria and viruses regarding water systems/water pipes, FLA diversity is not payed sufficient attention. Most of the FLA are able to form cysts (multi-walled structures) to withstand adverse environmental conditions and to be dispersed. This non active cyst provides a high tenacity and enables the cyst forming FLA to survive stress conditions such as desiccation or disinfection measures.

FLA are not obligatory parasites, they do not need a host to survive or proliferate, but several species or strains can live parasitically in humans and animals [4]. They cause different diseases with different clinical presentations which may affect the central nervous system, the eyes, or the skin of humans.

In addition to their relevance as human pathogens, a range of different FLA (and amoebal stages of slime moulds) are known to act as reservoirs and carriers of other pathogenic microorganisms, hereby protecting them and interacting with them to a high degree [5].

\section{FLA as Human pathogens}

Several FLA are known to be pathogenic for humans and animals. Some Acanthamoeba strains, and the two FLA-species Balamuthia mandrillaris and Naegleria fowleri are well known as pathogens for many years (for review see: [6-8]). They can generate diseases in humans when they get in close contact with humans, e.g., while swimming in unchlorinated pools or while wearing contact lenses. Naegleria fowleri is the etiological agent of an acute, progressive encephalitis, the so-called Primary Amebic Meningoencephalitis (PAM(E)), affecting young immunocompetent (and otherwise healthy) people. Several Acanthamoeba strains are responsible for the so-called Granulomatous Amebic Encephalitis (GAE), a rare chronic disease, that arises particularly in immunocompromised people. Acanthamoeba keratitis (AK) is predominantly associated with wearing contact lenses. These AKcases, affecting immunocompetent people, have been more frequent in recent years while human GAE cases caused by Balamuthia mandrillaris (notably in immunocompromised people) are rare.

\subsection{Acanthamoebae}

Acanthamoebae are the most commonly detected FLA in the environment. They have a two stage life cycle which includes a vegetative form (trophozoite) as a motile trophic stage (Figure 1), when the amoeba is in a humid, favorable and nutrition rich environment. When not adherent to a surface, they can also form so called swimming-forms showing their typical acanthapodia (Figure 2). They become dormant and enter the cyst stage (Figure 3) as soon as the conditions become not favorable.

As cysts, the Acanthamoebae are resistant to a certain degree to environmental or man-made stress (e.g., disinfection measures or medical treatment). They are able to tolerate a wide range of environmental conditions. As some Acanthamoeba strains are thermotolerant, they are capable of infecting humans.

The virulence of the Acanthamoebae varies among their species and strains. Currently Acanthamoebae are attributed to genotypes/sequence types, based on their nuclear small-subunit ribosomal RNA (rRNA) gene. T4 is the predominant genotype in nature and in association with patients, while others have also been proven to be pathogenic [9-11]. As trophozoites they adhere to host cells and trigger several symptoms of the Acanthamoebiasis. The progressive ocular diseaseAcanthamoeba keratitis (AK) - most frequently affects immunocompetent hosts, most of them being (soft) contact lens wearers (for case report see: [12]). Improper handling of the contact lenses and the development of biofilm within the lens cases may lead to AK, a sight- threatening corneal infection. 
Among the risk factors, patient behavioral factors are among the most prominent and important factors, e.g., wearing contact lenses while swimming, diving or bathing in non-chlorinated pools. Cutaneous infections are also rare manifestations of an Acanthamoebiasis (for review see: [6]).

Some strains of the genus Acanthamoeba are the etiologic agents of Granulomatous Amoebal (meningo-) Encephalitis (GAE). This CNS amoebiasis can be subacute to chronic, showing symptoms such as headache, neck stiffness, seizures, memory disorders and hemipareses. GAE is characterized by granular, focal brain lesions leading to brain necrosis and edema. Cysts can be found in the brain tissue and cerebrospinal fluid. Patients with a weakened immune system or suffering from chronic diseases are the most commonly affected. Acanthamoeba trophozoites (Figure 1) can reach the brain via the olfactory neural epithelium. Consequently they are dispersed hematogenously to the central nervous system [6].

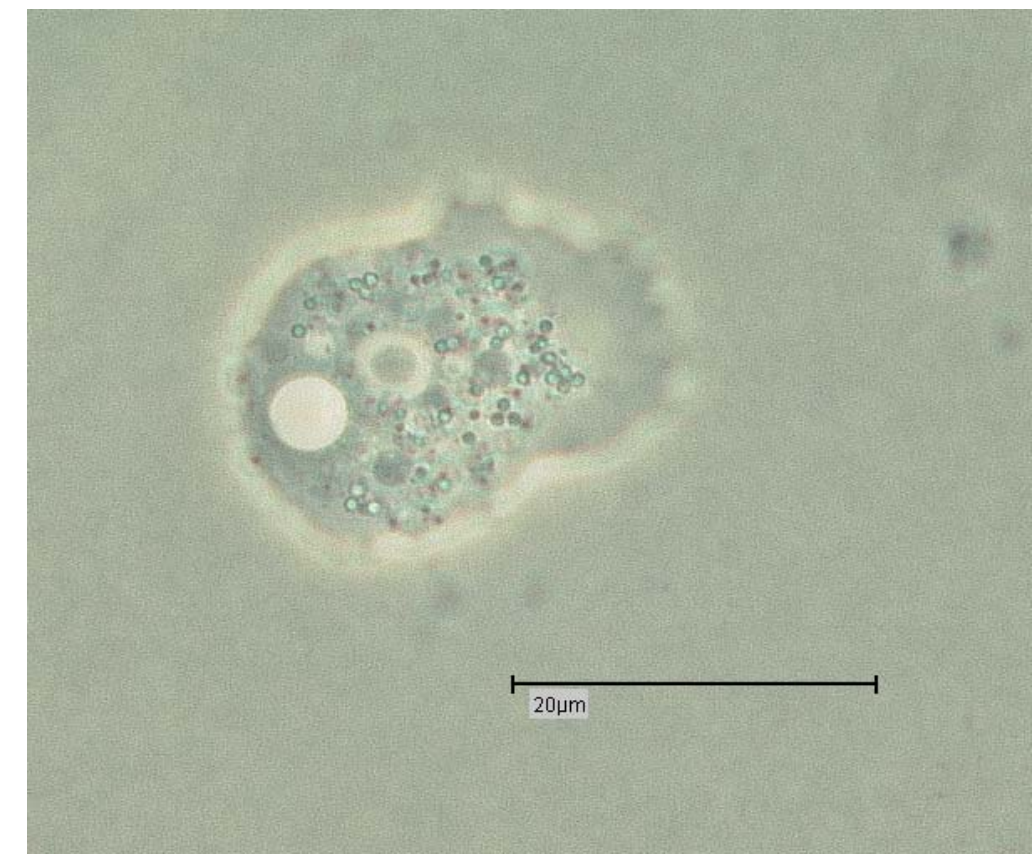

Figure 1. Adherent trophozoite of Acanthamoeba sp.; Light microscopy; phase contrast; Bar: $20 \mu \mathrm{m}$ (photo: Mais).

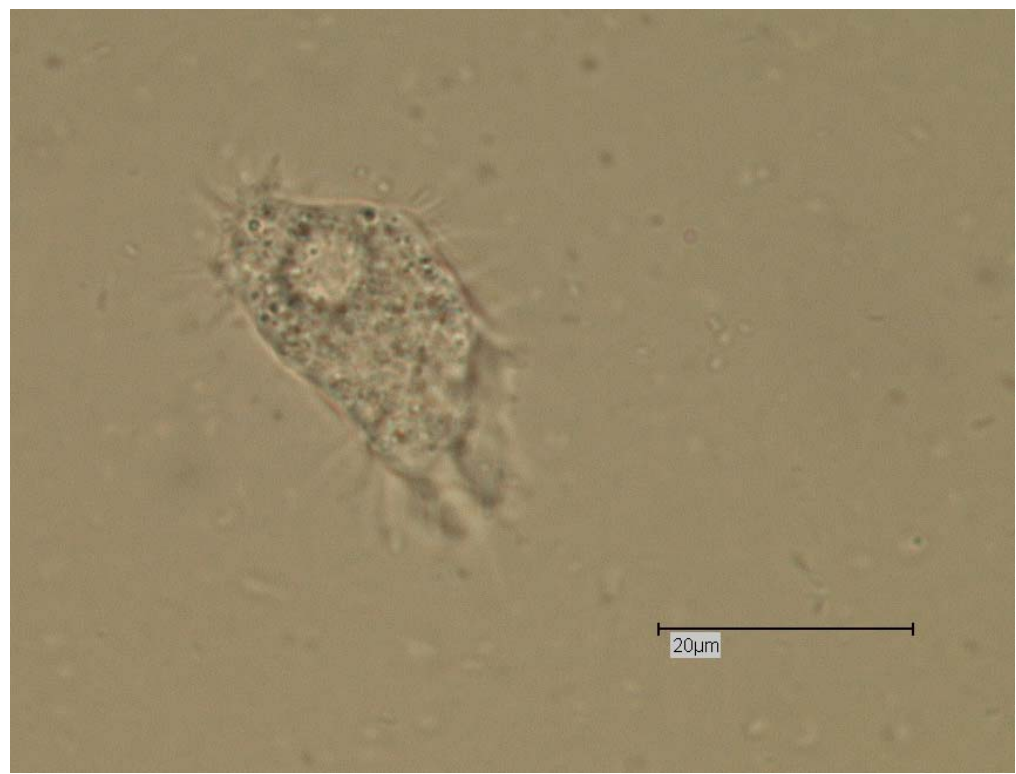

Figure 2. Floating form/swimming form of Acanthamoeba sp.; Light microscopy; phase contrast; Bar: $20 \mu \mathrm{m}$. 


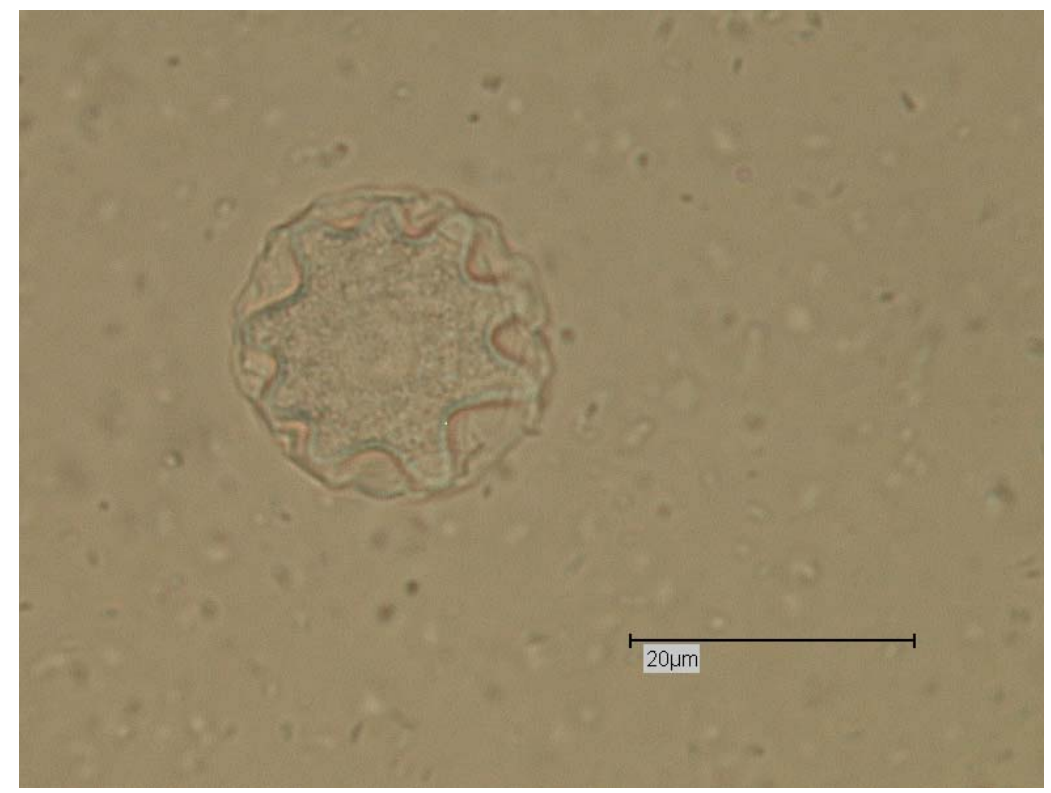

Figure 3. Cyst of Acanthamoeba sp.; light microscopy; phase contrast; Bar: $20 \mu \mathrm{m}$.

\subsection{Naegleria fowleri}

Naegleria amoebae are naturally to be found in humid soils and freshwater bodies but also in artificially heated waters, fountains, spas, and industrial cooling waters. Beside the trophozoite (the amoeboid, motile form; Figure 4) and the cyst stage (Figure 4) there is a third stage, as Naegleria has the potential of transforming into an amoeba-flagellate (Figure 5) under certain environmental conditions.

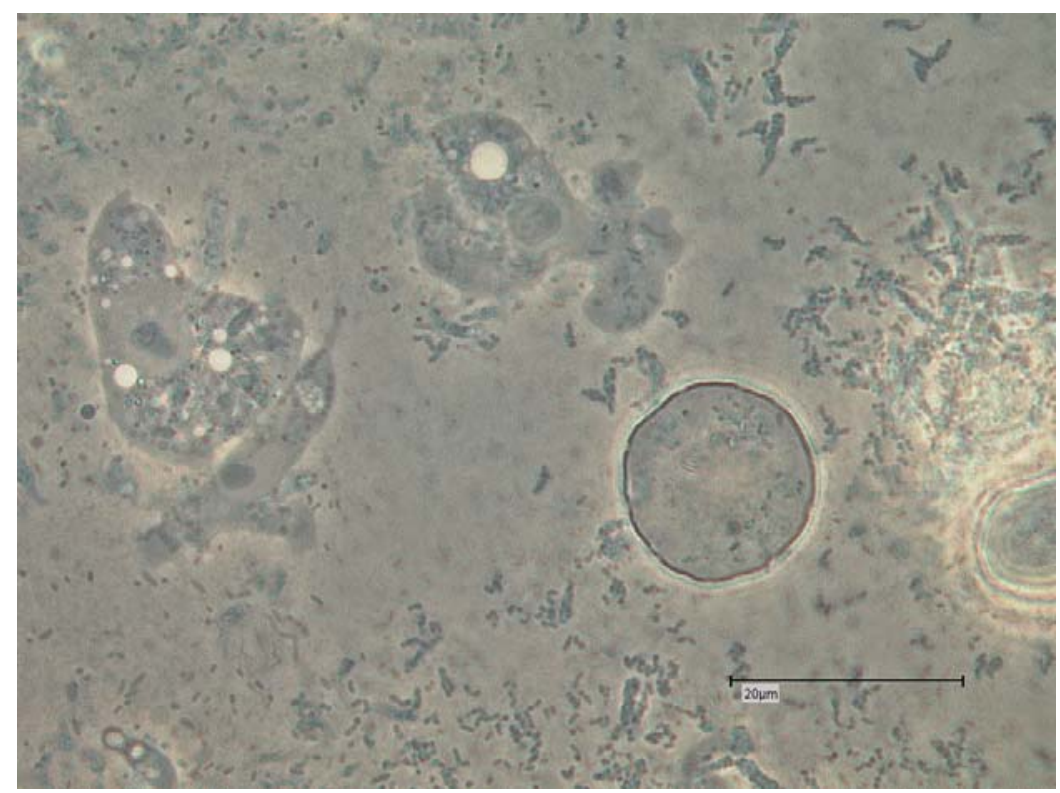

Figure 4. Trophozoite and cyst of Naegleria sp.; Light microscopy; phase contrast; Bar: $20 \mu \mathrm{m}$. 


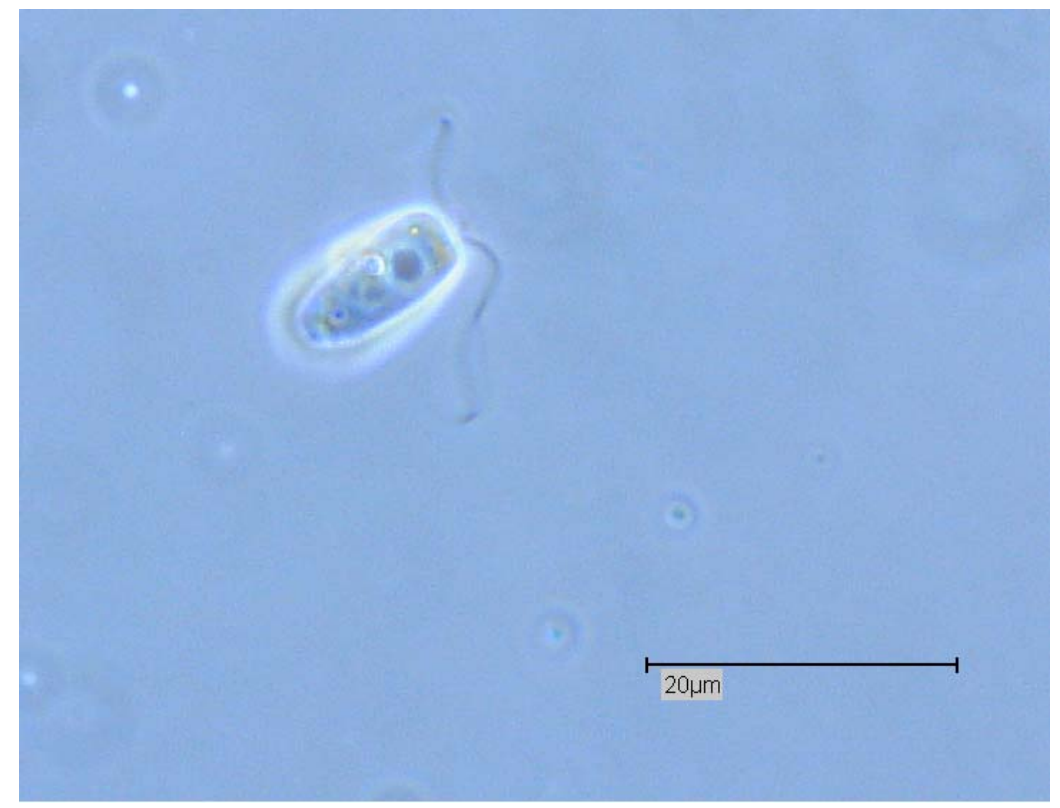

Figure 5. Flagellate stage of Naegleria sp.; Light microscopy; phase contrast; Bar: $20 \mu \mathrm{m}$.

Naegleriasis is a rare but often fatal disease, which is caused by Naegleria fowleri. To date, the species $N$. fowleri is known in humans as the only etiological agent. The infection affects healthy children and young adults after they have had activities in warm freshwater lakes and ponds. These "brain eating amoebae" have recently gained public attention because several healthy young people have shown a rapid onset of symptoms following exposure to fresh water and have died in the USA (e.g., Arizona) after short incubation periods $[13,14]$. N. fowleri trophozoites reach the brain migrating along the olfactory nerve [15]. The clinical picture of this central nervous system disease presents with tissue damages as a diffuse meningoencephalitis, often with a fatal outcome [13,14]. The disease is therefore called Primary Amebic Meningoencephalitis (PAM(E)) (for review see: [7]). N. fowleri is thermotolerant and survives temperatures of $45{ }^{\circ} \mathrm{C}$. $\operatorname{PAM}(\mathrm{E})$ is characterized by headache, neck stiffness and a very acute progression.

\subsection{Balamuthia mandrillaris}

Balamuthia mandrillaris is a free living amoeba with two stages, a motile trophozoite and a cyst stage, and the cyst stage provides high tenacity. It can be found in soil, dust and water. It can cause a GAE, the so called Granulomatous Balamuthia Amebic Encephalitis (also: BAE), showing up with neck stiffness and headache. DNA of $B$. mandrillaris has been found in a contact lens case during routine investigation for AK [16]. Cutaneous infections have also been described in both immunocompetent and immunocompromised persons (for review see: [8]).

\subsection{Other FLA with Pathogenic Capability Discovered to Date}

FLA of the genus Sappinia cause the so-called Sappinia Amebic Encephalitis (SAE). Sappiniae are usually found in soil in the natural environment. The species Sappinia pedata is believed to be the etiolgical agent in one human case so far [17].

Hartmannella sp. has been associated with keratitis cases. It remains controversial whether Hartmannella (resp. Vermamoeba) strains may have been the etiological agent or just contaminants [18].

Vahlkampfiae were also detected, together with Hartmannellae while investigating a keratitis of a contact lens wearer (19). Paravahlkampfia francinae (n. sp.) has been found in the cerebrospinal fluid of a patient with PAM(E)-like symptoms [20].

Allovahlkampfia spelae was just recently identified as the etiological agent of a human keratitis [21].

Dictyostelium polycephalum, a social amoeba (slime mold; belonging to the Mycetozoa), was also involved in a keratitis case of an immunocompetent patient [22]. Similar to the FLA trophozoites, $D$. 
polycephalum has a unicellular amoebal vegetative stage which is phagocytosis active (Figure 6). A multicellular fruiting body can be developed (Figure 7).

With an increase in the FLA research further FLA genera, species, or strains will be detected in the future, possibly affecting the health of humans or animals directly as opportunistic parasites. The microbiological investigation of drinking water (tap water, domestic water) still relies on bacteria and viruses rather than FLA. While being very abundant and hiding in biofilms, these pathogenic FLA should be considered as important water-derived human parasites in future.

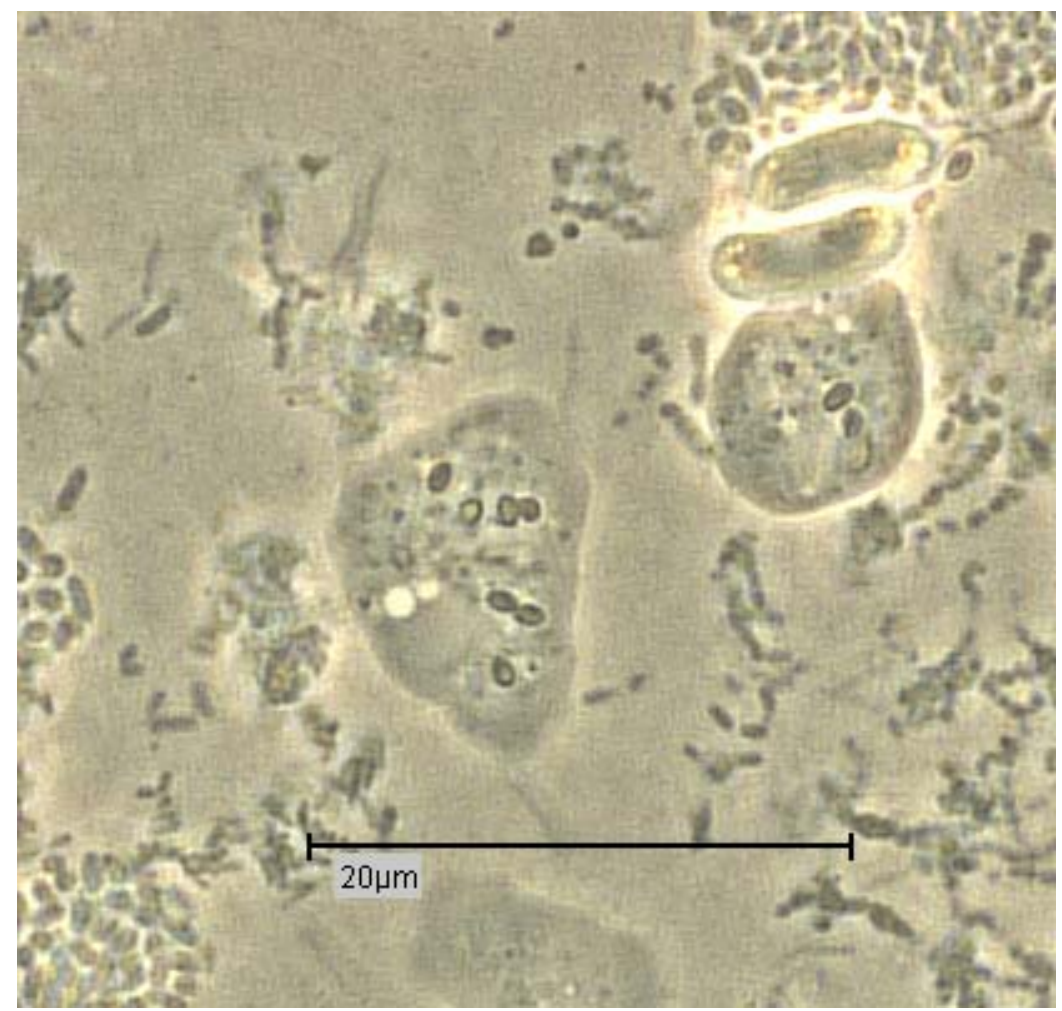

Figure 6. Amoebal stages of Dictyostelium sp.; Light microscopy; Bar: $20 \mu \mathrm{m}$ (photo: Mais).

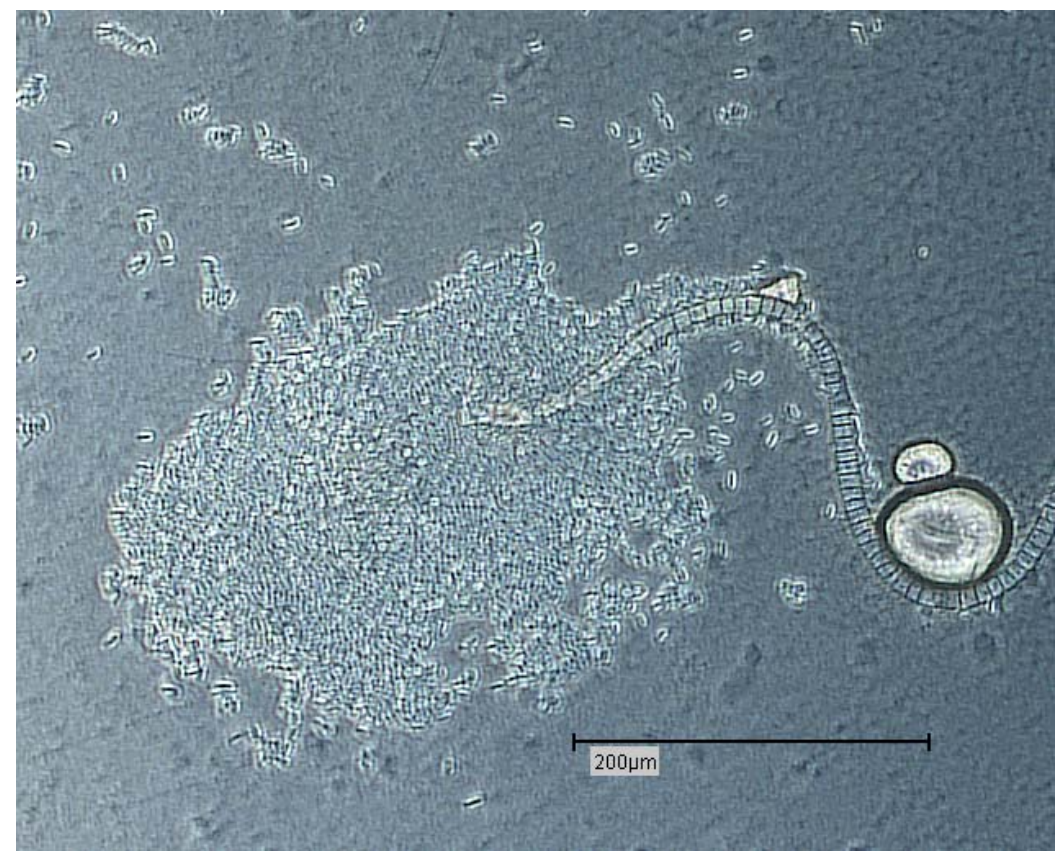

Figure 7. Fruiting body of Dictyostelium sp.; Sporangium and Sporangophore; Light microscopy; phase contrast; Bar: $50 \mu \mathrm{m}$ (photo: Mais). 


\section{FLA as Reservoirs for Human Pathogens}

Waterborne pathogenic microorganisms are well-known to cause disease in individuals or outbreaks with large patient numbers by contaminating community water supplies. As trophozoites the FLA feed as heterotrophic predators on other microorganisms within their biocoenoses. There have been numerous examples throughout the years of (water-borne) microorganisms (e.g., bacteria) being taken up by FLA via phagocytosis but resisting complete digestion. Some of these microorganisms not only survived intracellularly and escaped lysis but also proliferated inside the amoebal host (for review see: [5]. The FLA therefore may act as reservoirs for diverse microorganisms, among them pathogenic microorganisms with a Public Health and Environmental Health potential. These intracellularly surviving and/or proliferating microorganisms are called endocytobionts [5]. In Table 1 an overview of selected bacterial endocytobionts with pathogenic potential or Public Health relevance is given (in situ and in vitro studies).

Table 1. Examples for bacterial endocytobionts with Public Health relevance associated with FLA.

\begin{tabular}{ll}
\hline Bacterial Endocytobionts & FLA as Hosts \\
\hline Staphylococcus aureus (incl.MRSA) & Acanthamoebae \\
Listeria monocytogenes & Acanthamoebae \\
Bacillus anthracis & Acanthamoeba castellanii; Hartmannella (Vermamoeba) vermiformis \\
Escherichia coli O157 & Acanthamoebae \\
Salmonella spp. & Acanthamoebae \\
Yersinia enterocolitica & Acanthamoebae \\
Vibrio cholerae & Acanthamoebae (Acanthamoeba castellanii) \\
Campylobacter sp. & Acanthamoebae (Acanthamoeba polyphaga) \\
Helicobacter pylori & Acanthamoebae \\
Legionella pneumophila & Acanthamoebae \\
\hline
\end{tabular}

The most-known endocytobiont of FLA ist certainly Legionella pneumophila, the etiological agent of Legionaires' disease [23-26]. L. pneumophila is confirmed to proliferate inside FLA including Acanthamoeba spp., Naegleria spp. and Hartmannella spp. [5] (see Table 1). Following the uptake of the free legionellae by a trophozoite, the legionellae escape into the amoebal cytoplasm and are therefore not lysed. The intracellular replication is temperature dependent. If they succeed in surviving the uptake, and evade digestion, the legionellae lyse the amoebal host, to be released in the environment. Furthermore this passage through the amoebal trophozoites without being digested reactivates "viable but non culturable" (VBNC) legionellae, as typically found in biofilms. The pathogenicity and the infectivity of Legionellae for humans is increased significantly after the amoebal passage $[27,28]$. The dispersal of mycobacteria in the environment and water distribution systems is enhanced by FLA. Mycobacterium avium (associated with respiratory disease) multiplies intracellularly in Acanthamoeba castellanii. These mycobacteria show an enhanced virulence following the amoebal passage. Listeria monocytogenes, Campylobacter jejuni, Arcobacter butzleri, Vibrio cholera, Yersinia enterocolitica or Salmonella typhimurium (see Table 1) are further examples of pathogenic endocytobionts in Acanthamoeba [29-31]. Pseudomonas aeruginosa, Simkania negevensis, Chlamydia- and Ehrlichia-like organisms, Burkholderia pseudomallei, Escherichia coli and Francisella tularensis are also associated with FLA (table 1 is extracted from [31]). Those bacteria are protected against adverse conditions, especially when hiding in cyst forming FLA $[32,33]$. They show increased resistance to biocides. Hence, pathogenic bacteria internalized in amoebae represent a high risk for Public Health because they are not easily reached by conventional biocide treatment (e.g., chlorine).

In addition to their role as reservoirs of bacteria, FLA play a role in the transport and proliferation of pathogenic viruses that are found in surface waters and in groundwater contaminated by (fecal) contamination. Polioviruses, Adenoviruses, and Enteroviruses (e.g., Coxsackieviruses) are transported and protected by FLA [34,35]. The association between FLA and giant viruses includes the Acanthamoeba polyphaga Mimivirus (APMV) (Figure 8) which turned out to be pathogenic in hospital-acquired pneumonia [36]. Although the so called Pandoraviruses (Figure 9) 
might be not relevant to humans in terms of pathogenicity, it has been shown how easily theses giant viruses can get in close contact with humans [37,38].

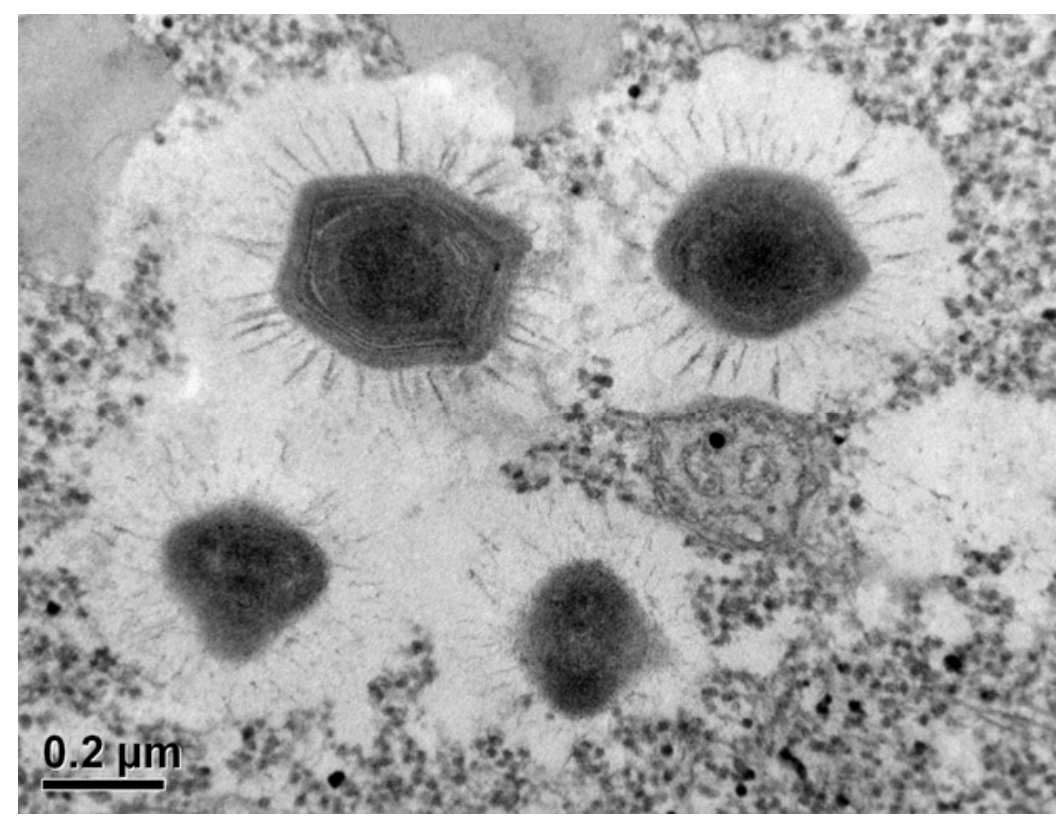

Figure 8. Acanthamoeba sp. trophozoite with intracellular (endocytobionts) Mimivirus (APMV) virions; Electron Microscopy (photo: Basoglu; Goethe university; Frankfurt; courtesy of Prof. Dr. Klimpel and Dr. Kuhn); Bar: $0.2 \mu \mathrm{m}$.

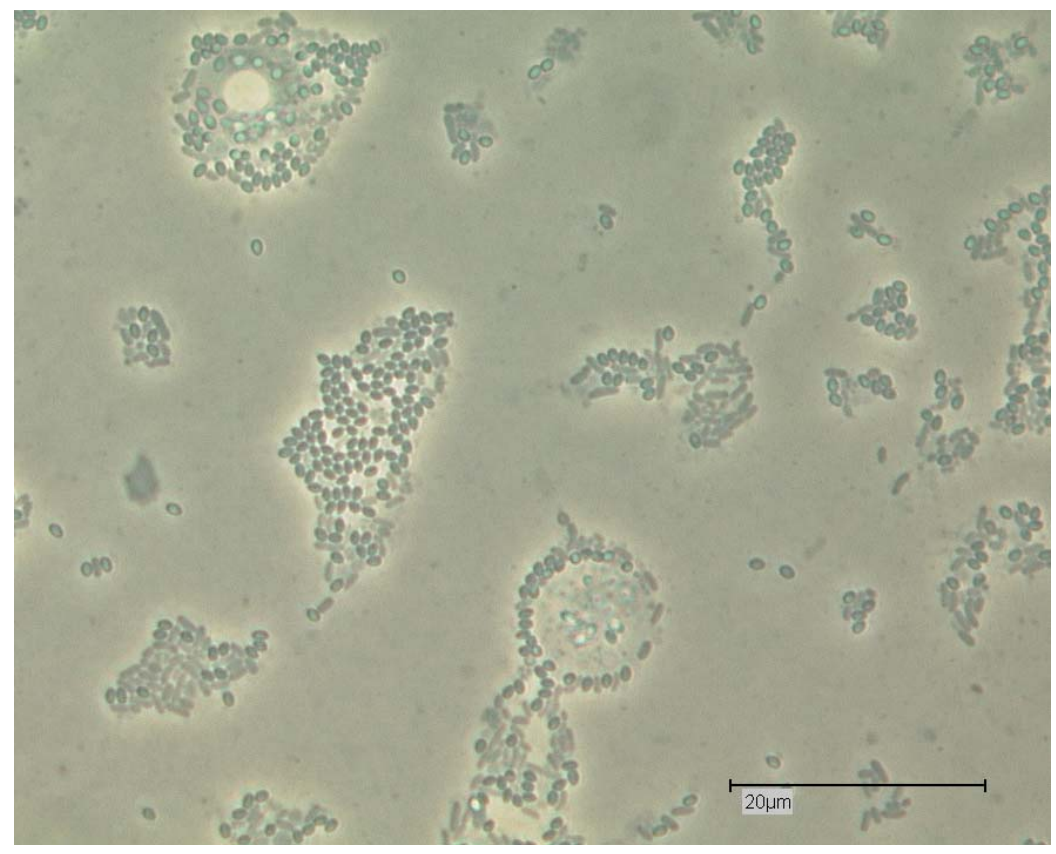

Figure 9. Acanthamoeba sp. trophozoites with Pandoravirus inopinatum virions; light microscopy; phase contrast; Bar: $20 \mu \mathrm{m}$.

The research focusing on the gene transfer of FLA, their viral endocytobionts and their simultaneously present bacterial endocytobionts, seems of particular interest when focusing on genome evolution. Beside those bacterial and viral endocytobionts, there are also pathogenic fungi (e.g., Cryptococcus neoformans; Histoplasma capsulatum) and protozoa (Toxoplasma gondii; Cryptosporidium spp.) which live in close association with FLA showing similar effects after the amoebal passage [31,39-41]. 
In conclusion FLA pose a relevance for the dispersal of pathogenic endocytobionts. Additionally FLA are of particular relevance for the protection of endocytobionts (especially in cyst forming FLA, in which the cyst is used as a protective shell). Another relevance includes the development and enhancement of (human) pathogenicity and virulence. A whole range of microorganisms uses FLA as "training grounds" or "test organisms" for their adaptation to life within human or animal cells (e.g., macrophages, which are very similar to amoebal trophozoites) [42-44]. The virulence of bacterial and fungal endocytobionts is enhanced, and the resistance against antibiotics or disinfection measures is increased after amoebal passage. These "FLA-endocytobiont" relationships can be regarded as evolutionary precursors of pathogenicity. Generally, global warming as a result of climate change could potentially turn the world to a more suitable habitat for parasites including FLA. Rising environmental temperatures might increase the frequency of "FLA-Human" contact, shifting towards parasitic (or clinically relevant) interactions or transmission of endocytobionts. The fact that FLA also serve as hosts of and vehicles (vectors) for pathogenic microorganisms has an impact on the Public Health sector, affecting the quality of water. Therefore, FLA should definitely be considered in the context of Public Health as water-borne parasites, protective shells and vehicles for pathogenic microorganisms in water networks.

Acknowledgments: The author would like to thank David Lam (MD, MPH, Shaman Medical Consulting) for review and English language editing of the article. Further thanks go to the contributors of the valuable photos.

Conflicts of Interest: The author declares no conflict of interest.

\section{References}

1. Trabelsi, H.; Dendana, F.; Sellami, A.; Sellami, H.; Cheikhrouhou, F.; Neji, S.; Makni, F.; Ayadi, A. Pathogenic free-living amoebae: Epidemiology and clinical review. Pathol. Biol. 2012, 60, 399-405.

2. Ashbolt, N. Environmental (saprozoic) pathogens of engineered water systems: Understanding their ecology for risk assessment and management. Pathogens 2015, 4, 390-405.

3. Borde, J.; Helwig, P.; Hauschild, O. Gelenkprothesen-Infektionen. Krankenhaushygiene up2date 2013, 8, 89-97.

4. Scheid, P. Free-Living Amoebae (FLA), Introduction. Encyclopedia of Parasitology; Mehlhorn, H., Ed.; Springer: Berlin/Heidelberg, Germany, 2016; pp. 1074-1076, doi:10.1007/978-3-662-43978-4_3465.

5. Scheid, P. Relevance of free-living amoebae as hosts for phylogenetically diverse microorganisms. Parasitol. Res. 2014, 113, 2407-2414.

6. Scheid, P. Free-Living Amoebae as Human Pathogens: (Genus) Acanthamoeba. In Encyclopedia of Parasitology; Mehlhorn, H., Ed.; Springer: Berlin/Heidelberg, Germany, 2016; doi:10.1007/978-3-662-43978-4_8.

7. Scheid, P. Free-Living Amoebae as Human Pathogens: Naegleria fowleri. In Encyclopedia of Parasitology; Mehlhorn, H., Ed.; Springer: Berlin/Heidelberg, Germany, 2016; pp. 1082-1083, doi:10.1007/978-3-66243978-4_2060.

8. Scheid, P. Free-Living Amoebae as Human Pathogens: Balamuthia mandrillaris. In Encyclopedia of Parasitology; Mehlhorn, H., Ed.; Springer: Berlin/Heidelberg, Germany, 2016; pp. 1080-1081, doi:10.1007/978-3-662-43978-4_354.

9. Booton, G.; Visvesvara, G.; Byers, T.; Kelly, D.; Fuerst, P. Identification and distribution of Acanthamoeba species genotypes associated with nonkeratitis infections. J. Clin. Microbiol. 2005, 43, 1689-1693.

10. Qvarnstrom, Y.; Nerad, T.; Visvesvara, G. Characterization of a new pathogenic Acanthamoeba species, A. byersi n. sp., isolated from a human with fatal amoebic encephalitis. J. Eukaryot. Microbiol. 2013, 60, 626-633.

11. Grün, A.-L.; Stemplewitz, B.; Scheid, P. First report of an Acanthamoeba genotype T13 isolate as etiological agent of a keratitis in humans. Parasitol. Res. 2014, 113, 2395-2400.

12. Scheid, P.; Zoller, L.; Pressmar, S.; Richard, G.; Michel, R. An extraordinary endocytobiont in Acanthamoeba sp.isolated from a patient with keratitis. Parasitol. Res. 2008, 102, 945-950.

13. Martinez, A.J.; Visvesvara, G.S. Free-living, amphizoic and opportunistic amebas. Brain Pathol. 1997, 7, $583-598$.

14. Marciano-Cabral, F.; Cabral, G.A. The immune response to Naegleria fowleri amebae and pathogenesis of infection. FEMS Immunol. Med. Microbiol. 2007, 51, 243-259.

15. Visvesvara, G. Free-living amebae as opportunistic agents of human disease. J. Neuroparasitol. 2010, 1, N100802, doi:10.4303/jnp/N100802. 
16. Balczun, C.; Scheid, P. Detection of Balamuthia mandrillaris DNA in the storage case of contact lenses in Germany. Parasitol. Res. 2016, 115, 2111-2114.

17. Qvarnstrom, Y.; da Silva, A.; Schuster, F.; Gelman, B.; Visvesvara, G. Molecular confirmation of Sappinia pedata as a causative agent of amoebic encephalitis. J. Infect. Dis. 2009, 199, 1139-1142.

18. De Jonckheere, J.; Brown, S. Non-Acanthamoeba amoebic keratitis. Cornea 1999, 18, 499-501.

19. Aitken, D.; Hay, J.; Kinnear, F.; Kirkness, C.; Lee, W.; Seal, D. Amebic keratitis in a wearer of disposable contact lenses due to a mixed Vahlkampfia and Hartmannella infection. Ophthalmology 1996, 103, 485-494.

20. Visvesvara, G.; Sriram, R.; Qvarnstrom, Y.; Bandyopadhyay, K.; da Silva, A.; Pieniazek, N.; Cabral, G. Paravahlkampfia francinae n. sp. masquerading as an agent of primary amoebic meningoencephalitis. J. Eukaryot. Microbiol. 2009, 56, 357-366.

21. Tolba, M.; Huseein, E.; Farrag, H.; Mohamed, H.; Kobayashi, S.; Suzuki, J.; Ali, T.; Sugano, S. Allovahlkampfia spelaea causing keratitis in humans. PLoS Negl. Trop. Dis. 2016, 10, e0004841.

22. Reddy, A.; Balne, P.; Garg, P.; Sangwan, V.; Das, M.; Krishna, P.; Bagga, B.; Vemuganti, G. Dictyostelium polycephalum infection of human cornea. Emerg. Infect. Dis. 2010, 16, 1644-1645.

23. Rowbotham, T. Preliminary report on the pathogenicity of Legionella pneumophila for freshwater and soil amoebae. J. Clin. Pathol. 1980, 33, 1179-1183.

24. Boulanger, C.A.; Edelstein, P.H. Precision and accuracy of recovery of Legionella pneumophila from seeded tap water by filtration and centrifugation. Appl. Environ. Microbiol. 1995, 61, 1805-1809.

25. Hägele, S.; Kohler, R.; Merkert, H.; Schleicher, M.; Hacker, J.; Steinert, M. Dictyostelium discoideum: A new host model system for intracellular pathogens of the genus Legionella. Cell. Microbiol. 2000, 2, 165-171.

26. Hookey, J.; Saunders, N.; Fry, N.; Birtles, R.; Harrison, T. Phylogeny of Legionellaceae based on smallsubunit ribosomal DNA sequences and proposal of Legionella lytica comb. nov. for Legionella-like amoebal pathogens. Int. J. Syst. Bacteriol. 1996, 46, 526-531.

27. Bruggemann, H.; Hagman, A.; Jules, M.; Sismeiro, O.; Dillies, M.-A.; Gouyette, C.; Kunst, F.; Steinert, M.; Heuner, K.; Coppee, J.-Y.; et al. Virulence strategies for infecting phagocytes deduced from the in vivo transcriptional program of Legionella pneumophila. Cell. Microbiol. 2006, 8, 1228-1240.

28. Cirillo, J.; Falkow, S.; Tompkins, L.; Bermudez, L. Interaction of Mycobacterium avium with environmental amoebae enhances virulence. Infect. Immun. 1997, 65, 3759-3767.

29. Snelling, W.; McKenna, J.; Lecky, D.; Dooley, J. Survival of Campylobacter jejuni in waterborne protozoa. Appl. Environ. Microbiol. 2005, 71, 5560-5571.

30. King, C.; Shotts, E., Jr.; Wooley, R.; Porter, K. Survival of coliforms and bacterial pathogens within protozoa during chlorination. Appl. Environ. Microbiol. 1988, 54, 3023-3033.

31. Balczun, C.; Scheid, P. Free-Living Amoebae as Hosts for and Vectors of Intracellular Microorganisms with Public Health Significance. Viruses 2017, 9, 65, doi:10.3390/v9040065.

32. Greub, G.; Raoult, D. Microorganisms resistant to free-living amoebae. Clin. Microbiol. Rev. 2004, 17, $413-433$.

33. Wieser, A.; Schubert, S. Intra-und extrazelluläre Biofilme uropathogener E. coli. Chemother. J. 2011, 20, 181-185.

34. Scheid, P.; Schwarzenberger, R. Acanthamoeba spp. as vehicle and reservoir of adenoviruses. Parasitol. Res. 2012, 111, 479-485.

35. Mattana, A.; Serra, C.; Mariotti, E.; Delogu, G.; Fiori, P.L.; Cappuccinelli, P. Acanthamoeba castellanii promotion of in vitro survival and transmission of coxsackie b3 viruses. Eukaryot. Cell 2006, 5, 665-671.

36. Aherfi, S.; Colson, P.; La Scola, B.; Raoult, D. Giant viruses of amoebas: An update. Front. Microbiol. 2016, 7, 349.

37. Scheid, P.; Balczun, C.; Schaub, G.A. Some secrets are revealed: Parasitic keratitis amoebae as vectors of the scarcely described pandoraviruses to humans. Parasitol. Res. 2014, 113, 3759-3764.

38. Antwerpen, M.; Georgi, E.; Zoeller, L.; Woelfel, R.; Stoecker, K.; Scheid, P. Whole-genome sequencing of a Pandoravirus isolated from keratitis-inducing Acanthamoeba. Genome Announc. 2015, 3, e00136-15.

39. Steenbergen, J.; Shuman, H.; Casadevall, A. Cryptococcus neoformans interactions with amoebae suggest an explanation for its virulence and intracellular pathogenic strategy in macrophages. Proc. Natl. Acad. Sci. USA 2001, 98, 15245-15250.

40. Scheid, P.; Schwarzenberger, R. Free-living amoebae as vectors of cryptosporidia. Parasitol. Res. 2011, 109, 499-504.

41. Winiecka-Krusnell, J.; Dellacasa-Lindberg, I.; Dubey, J.P.; Barragan, A. Toxoplasma gondii: Uptake and survival of oocysts in free-living amoebae. Exp. Parasitol. 2009, 121, 124-131. 
42. Steinert, M.; Birkness, K.; White, E.; Fields, B.; Quinn, F. Mycobacterium avium bacilli grow saprozoically in coculture with Acanthamoeba polyphaga and survive within cyst walls. Appl. Environ. Microbiol. 1998, 64, 2256-2261.

43. Fritsche, T.; Sobek, D.; Gautom, R. Enhancement of in vitro cytopathogenicity by Acanthamoeba spp. following acquisition of bacterial endosymbionts. FEMS Microbiol. Lett. 1998, 166, 231-236.

44. Molmeret, M.; Horn, M.; Wagner, M.; Santic, M.; Kwaik, Y. Amoebae as training grounds for intracellular bacterial pathogens. Appl. Environ. Microbiol. 2005, 71, 20-28.

(C) 2018 by the authors. Licensee MDPI, Basel, Switzerland. This article is an open access article distributed under the terms and conditions of the Creative Commons Attribution (CC BY) license (http://creativecommons.org/licenses/by/4.0/). 